\section{En conclusion}

Diminuer les émissions de gaz à effet de serre reste la condition sine qua non pour limiter le réchauffement global. Depuis 1750, un total de plus de 2500 milliards de tonnes de $\mathrm{CO}_{2}$ (2 $500 \mathrm{GtCO}_{2}$ ) a déjà été émis. Les émissions cumulées de carbone compatibles avec les cibles de réchauffement envisagées dans l'Accord de Paris restent proches de celles estimées dans le rapport spécial SR1.5 (figure 2). Atteindre de tels objectifs représente un défi technique et civilisationnel d'autant plus important que la capacité des océans et des continents à piéger une fraction importante de nos émissions de $\mathrm{CO}_{2}$ diminuera au cours de la seconde moitié du $\mathrm{XXI}^{\mathrm{e}}$ siècle si nous n'agissons pas dès maintenant pour limiter les émissions de $\mathrm{CO}_{2}$. Il est également urgent de limiter les émissions de méthane, ce qui s'accompagnera d'une amélioration de la qualité de l'air et permettra de limiter le réchauffement qui résulterait d'une réduction de la pollution atmosphérique (souhaitable sur le plan sanitaire).

De tels efforts d'atténuation des émissions sont-ils encore possibles en temps voulu ? Ce n'est évidemment pas au premier groupe de travail du Giec de se prononcer à ce sujet. Mais force est de reconnaître qu'il ne suffit pas de décréter que c'est " physiquement et chimiquement possible " pour que cela se réalise. Ce n'est plus le scientifique, mais le citoyen lambda qui s'exprime ici : l'acceptabilité sociétale des mesures d'atténuation ne doit pas attendre que les politiques d'adaptation aux changements climatiques montrent leurs limites. Il s'agit donc pour le Giec d'informer au mieux les décideurs et l'ensemble des citoyens, afin de contribuer à une prise de conscience globale que le changement climatique n'est ni la fin du monde ni celle de l'humanité, mais bien la fin d'un monde où la consommation d'énergie était sans limite, les « biens communs » sans valeur économique et la préservation de l'environnement souvent confinée à quelques parcs nationaux et aires maritimes soi-disant protégées.

Hervé Douville Auteur coordinateur du chapitre 8, CNRM, Météo-France

\title{
« Destination 2030 » : innovation et impact à Eumetsat
}

Cet été dans l'hémisphère Nord a donné un aperçu sinistre de l'impact sur nos sociétés des phénomènes météorologiques extrêmes. Inondations, incendies et records de chaleur ont entraîné la mort de centaines de personnes, détruit des infrastructures et anéanti des activités économiques.

Dans le cadre de leur mission essentielle de protection des populations, les services météorologiques du monde entier sont confrontés à un défi de plus en plus urgent : transmettre au plus vite des informations précises sur les phénomènes météorologiques violents. La fréquence croissante de ces phénomènes découlant très probablement du changement climatique ne fait qu'ajouter à ce défi.

Eumetsat, l'agence européenne de satellites météorologiques, vient de publier une nouvelle stratégie à long terme baptisée «Destination 2030», qui vise précisément à apporter une réponse à ce défi (figure 1). Eumetsat prévoit de réaliser des progrès considérables au cours de la prochaine décennie en mettant en service des systèmes et des instruments satellitaires plus sophistiqués, en recourant à l'innovation dans des domaines tels que l'intelligence artificielle et l'apprentissage machine et en explorant les possibilités qu'offre le secteur du New Space ${ }^{1}$.

«Le tout premier objectif de cette stratégie, et le plus important, est de réussir le déploiement de systèmes

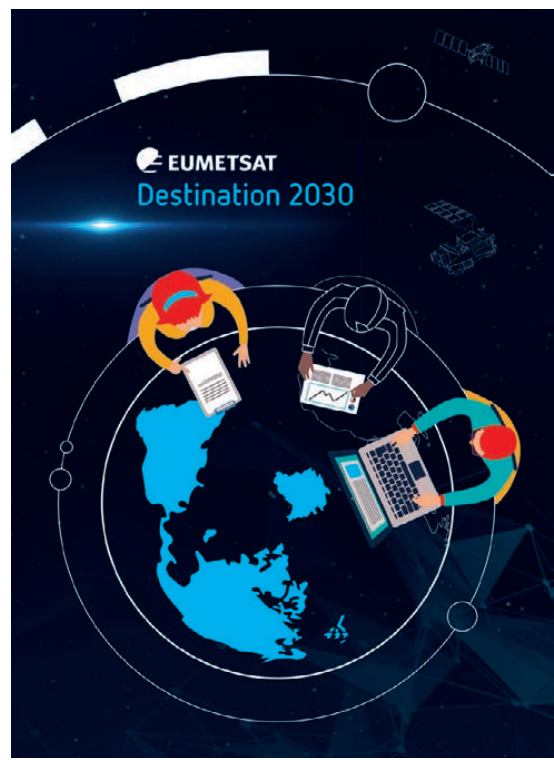

Figure 1. Couverture de la brochure « Destination 2030 " d'Eumetsat, accessible à l'adresse https://www.eumetsat.int/challenge-protectingagainst-extreme-weather-shapes-neweumetsat-strategy

satellitaires de nouvelle génération sur des orbites géostationnaires et polaires», indique Phil Evans, directeur général d'Eumetsat. "Notre système actuel en orbite polaire est déjà celui qui contribue le plus à la précision des prévisions météorologiques sur l'Europe à des échéances comprises entre 12 heures et 10 jours (voir par exemple Joo et al., 2013). Dans le même temps, notre système Meteosat en orbite géostationnaire se révèle essentiel à la prévision immédiate d'événements météorologiques à évolution rapide comme les fortes tempêtes. »

Les systèmes de nouvelle génération permettront d'atteindre un tout autre palier, en s'appuyant sur des instruments nouveaux et plus perfectionnés qui fourniront plus rapidement des volumes de données plus importants et de meilleure qualité.

«Pour ne citer qu'un exemple, les instruments à bord de nos satellites géostationnaires Meteosat Troisième Génération $(M T G)$ permettront, pour la première fois, de suivre le cycle de vie complet des orages. Ils détecteront l'instabilité initiale de l'atmosphère avant même que ne se forment des nuages, effectueront des observations plus rapides et à plus haute résolution de l'évolution et de la trajectoire des orages, et observeront les éclairs. Ce système va littéralement sauver des vies.»

Le lancement du premier satellite $M T G$ (figure 2) est prévu pour la fin 2022 et celui du premier satellite du Système polaire de seconde génération d'Eumetsat $(E P S-S G)$, en orbite basse, à la fin 2023. «D'ici 2030, Eumetsat aura renouvelé ses flottes sur les deux types d'orbites », ajoute Phil Evans.

1. Industrie spatiale émergente d'initiative privée, en particulier les nouvelles compagnies aérospatiales travaillant au développement d'un accès à bas coût et public à l'exploration de l'espace et aux technologies d'observation de la Terre. 
Tout le temps et partout, les nouveaux services de données d'EUMETSAT vous donnent accès à une mine de données

LES SERVICES

DE DONNÉES

D'EUMETSAT

- Rapides

- Reposant sur le cloud

- Précis

• Visualisations sur mesure

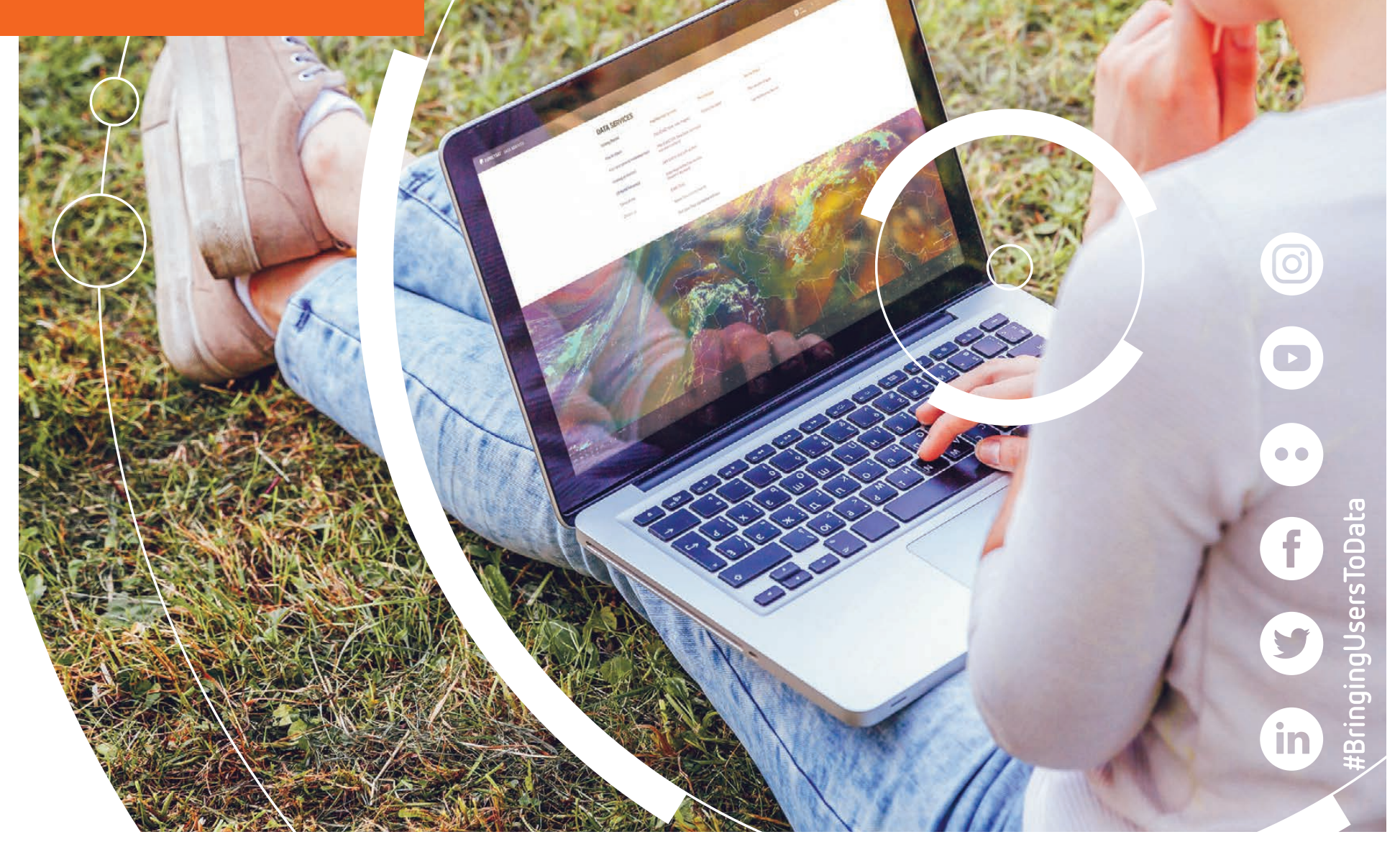


(a)

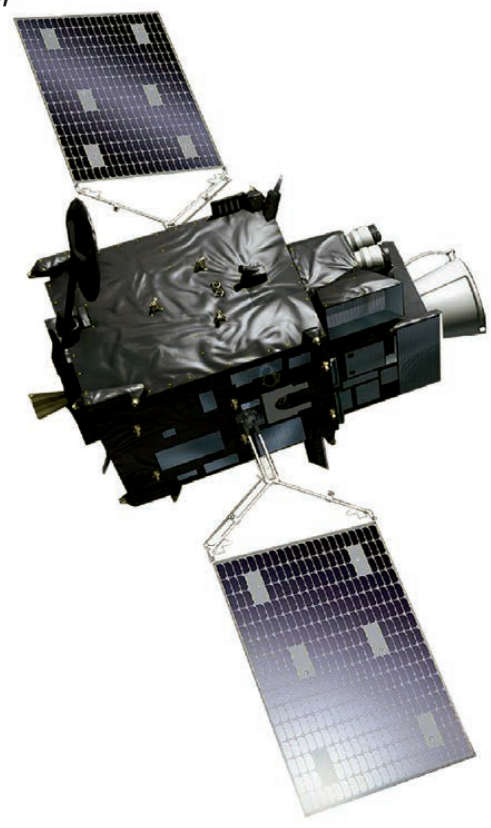

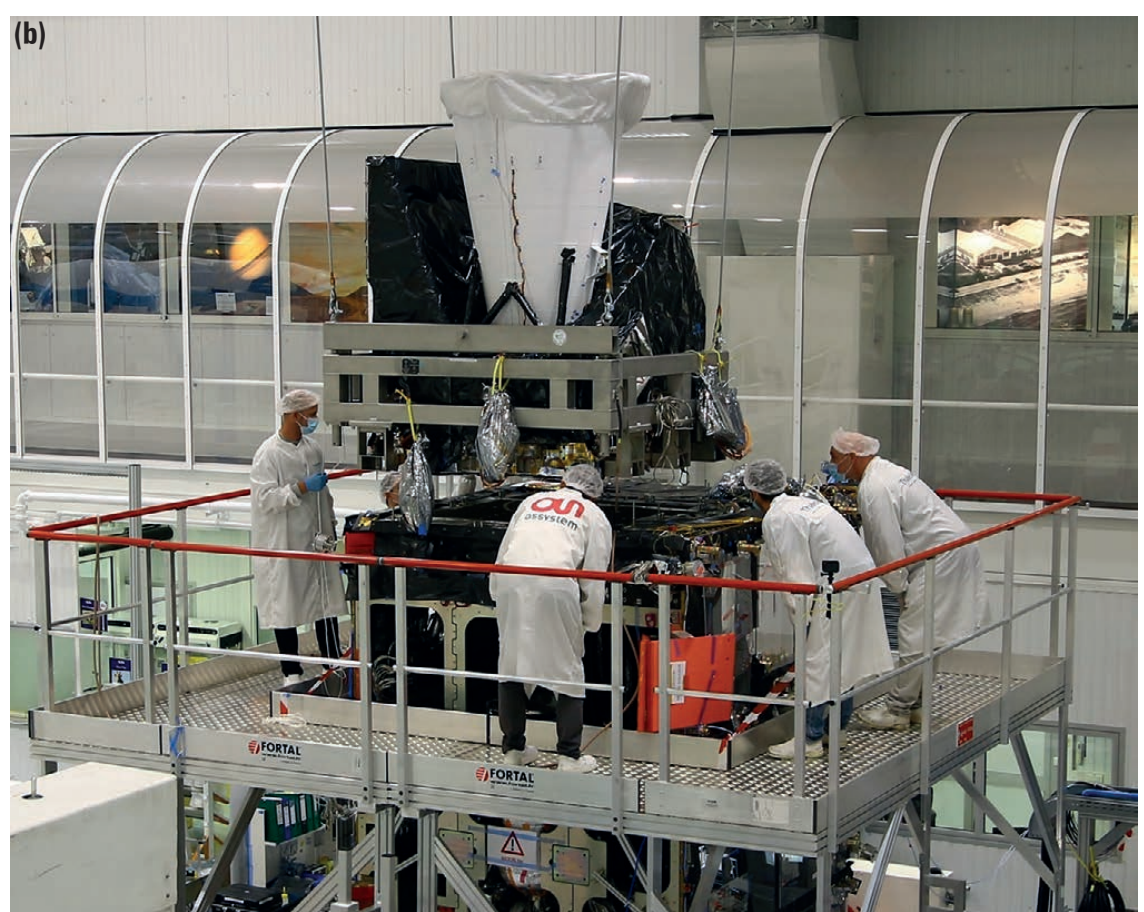

Figure 2. (a) Vue d'artiste du satellite MTG-I. (c) ESA. (b) Une fois terminé le premier modèle de vol de l'imageur FCl (Flexible Combined Imager), l'instrument a été monté avec succès sur la plateforme du prototype de vol de MTG-I. (C) Thales Alenia Space.

Avec Destination 2030, Eumetsat s'engage à continuer de miser sur l'innovation et la coopération afin de mettre à disposition plus rapidement et plus facilement de nouvelles données pour les prévisions météorologiques et climatologiques. "Cela suppose d'explorer les possibilités qu'offrent de nouvelles approches en matière de développement de satellites, y compris en examinant les avantages potentiels des innovations dans le domaine du New Space », précise Phil Evans. "Il s'agira aussi de trouver un équilibre approprié entre les approches traditionnelles et nouvelles.

Nous devons faire preuve d'innovation, tout en ayant conscience que ce sont nos grands satellites de référence qui resteront essentiels. Nous devons reconnaître que ce dont nous avons besoin, c'est de disposer de davantage d'informations de meilleure qualité et pas seulement de plus d'informations. De nouvelles entreprises spatiales explorent actuellement des pistes potentiellement très prometteuses, mais qui doivent être soigneusement évaluées et leurs impacts démontrés. Ce qui est crucial, c'est que les données que nous communiquons aux services météorologiques et hydrologiques de nos États membres contribuent de manière positive aux modèles de prévision météorologique.»
$\mathrm{Au}$ cœur de la stratégie «Destination $2030 »$ figure aussi le renforcement des efforts de coopération avec l'Union européenne et d'autres agences spatiales. "Forte de sa participation au programme d'observation de la Terre Copernicus de l'Union européenne et à des accords d'échange de données et d'informations scientifiques conclus avec d'autres agences spatiales, Eumetsat sera parfaitement en mesure de fournir des données globales très précises sur le système Terre, qui viendront alimenter les modèles météorologiques et climatologiques », poursuit Phil Evans.

«Les investissements que nous avons réalisés pour transposer des données en produits et en services utiles aux prévisions météorologiques et climatologiques, ainsi que dans les technologies cloud visant à rendre les accès à la fois plus rapides et plus faciles, continueront à porter leurs fruits. Afin de demeurer à la pointe en matière de gestion des données et de facilité d'accès des utilisateurs à celles-ci, Eumetsat a adopté une approche basée sur le cloud et les big data. »

«Permettez-moi de mettre cela en perspective. À la fin de l'année 2020, les archives d'Eumetsat, qui remontent à plus de 40 ans, enregistraient
6 pétaoctets ${ }^{2}$ de données. D'ici à la fin de l'année 2025, nous prévoyons que cette capacité atteindra environ 50 pétaoctets.

Nous estimons que nos nouveaux services ne profiteront pas seulement aux services météorologiques de nos États membres, mais qu'ils augmenteront considérablement la variété et le nombre d'utilisateurs de nos données. Je pense notamment au monde académique et à ceux qui souhaitent développer des applications liées à la météo ou au climat. Les objectifs énoncés dans Destination 2030 visent à doter les services météorologiques des États membres d'Eumetsat et d'autres pays du monde entier des données dont ils ont besoin pour protéger les populations concernées », explique Phil Evans. Et de conclure : "A l'heure où le dérèglement climatique affecte les phénomènes météorologiques, notre capacité à nous adapter à ces besoins est particulièrement importante.»

Isabelle Kling Eumetsat

\section{Un pétaoctet vaut un million de milliards} d'octets (ou $10^{15}$ octets). 